\section{Rheumatoid arthritis disease activity assessed by patient-reported outcomes and flow cytometry before and after an additional dose of COVID-19 vaccine}

The Centers for Disease Control and Prevention has recommended that moderately/severely immunosuppressed individuals receive an additional dose (AddDose) of COVID-19 vaccine at least 28 days after an initial mRNA vaccine series, or at least 2 months after a single adenovirus vector vaccine. ${ }^{1}$ Rheumatoid arthritis (RA) is frequently treated with immunosuppressive disease-modifying antirheumatic drugs (DMARDs), meaning that most patients with RA are eligible for an AddDose. The American College of Rheumatology suggests patients interrupt use of ('hold') certain DMARDs around the time of COVID-19 vaccination in attempt to boost immunogenicity. ${ }^{2}$ A small number of reports have noted no significant change in RA disease activity pre vaccination versus post vaccination against COVID19, but they assessed RA disease activity infrequently and did not compare results for patients who held versus continued DMARDs. Furthermore, data are lacking on the assessment of immune cellular populations possibly correlated with RA disease activity around the time of COVID-19 vaccination.

We conducted a prospective observational study of patients with RA treated at Brigham and Women's Hospital who were previously vaccinated against COVID-19 (two doses of mRNA vaccine or one dose of adenovirus vector vaccine). Subjects enrolled between July and November 2021, prior to receiving an AddDose. RA disease activity was assessed weekly using the validated patient-reported RA Disease Activity Index-5 (RADAI-5) from enrolment through 4 weeks post-AddDose via online data entry. ${ }^{3}$ Subjects completed an additional online survey 2 days after the AddDose regarding vaccine reactogenicity and whether they held or continued DMARDs. We compared mean RADAI-5 in the 4 weeks pre-AddDose versus the 4 weeks post-AddDose using the generalised estimating equation in SAS (V.9.4) to account for correlated data among individual subjects. We aimed to enrol 60 subjects to achieve $91 \%$ power to detect a $15 \%$ non-inferiority margin in RADAI-5 post-AddDose versus pre-AddDose.

Among 71 subjects, mean age was 62 years (SD 12) and 85\% were women. Methotrexate (42\%) and tumour necrosis factor inhibitors (38\%) were the most common DMARDs; 49\% held at least one DMARD (see table 1 for additional characteristics). Symptoms after the AddDose most often included injection site pain/swelling (72\%) and fatigue (52\%) (see online supplemental file 1). RADAI-5 was normally distributed pre-AddDose and post-AddDose. The mean RADAI-5 was 3.20 (SD 0.23) pre-AddDose compared with 3.25 (SD 0.23) post-AddDose (difference of $1.6 \%, \mathrm{p}=0.51$ ) (see online supplemental file 2). Figure 1 displays mean RADAI-5 in 35 (49\%) subjects who held DMARDs and 36 (51\%) subjects who continued all DMARDs. Mean RADAI-5 did not significantly differ pre-AddDose versus post-AddDose among subjects who held DMARDs, nor in subjects who continued all DMARDs. Mean change in RADAI-5 between pre-AddDose and post-AddDose did not significantly differ based on whether subjects held versus continued DMARDs ( $\mathrm{p}$ for interaction $=0.16$ ).

For a subset of 27 patients with seropositive RA, flow cytometry quantified percentages of 4 lymphocyte populations associated with immune activation in RA-T peripheral helper cells,
Table 1 Characteristics of study subjects

\begin{tabular}{|c|c|c|}
\hline & $\begin{array}{l}\text { All subjects } \\
(\mathrm{N}=71)\end{array}$ & $\begin{array}{l}\text { Subset of subjects } \\
\text { analysed by flow } \\
\text { cytometry }(\mathrm{N}=27)^{*}\end{array}$ \\
\hline Age, mean (SD) & $62.2(11.8)$ & $62.8(13.3)$ \\
\hline Female, $n(\%)$ & $60(84.5)$ & $20(74.1)$ \\
\hline White, $\mathrm{n}(\%)$ & $67(95.7) \dagger$ & $25(92.6)$ \\
\hline Seropositive rheumatoid arthritis, $\mathrm{n}(\%)$ & $62(87.3)$ & $27(100.0)$ \\
\hline \multicolumn{3}{|l|}{ Brand of initial vaccine series, $n(\%)$} \\
\hline Pfizer & $36(50.7)$ & $11(40.7)$ \\
\hline Moderna & $30(42.3)$ & $12(44.4)$ \\
\hline Johnson \& Johnson & $5(7.0)$ & $4(14.8)$ \\
\hline $\begin{array}{l}\text { Self-reported RA flare after initial } \\
\text { COVID-19 vaccine series, } \mathrm{n}(\%)\end{array}$ & $13(18.3)$ & $7(25.9)$ \\
\hline $\begin{array}{l}\text { COVID-19 infection prior to additional } \\
\text { dose, } n(\%)\end{array}$ & $1(1.4)$ & $0(0)$ \\
\hline $\begin{array}{l}\text { Mean (SD) days from completion of } \\
\text { initial vaccine series to additional dose }\end{array}$ & $177.8(48.1)$ & $184.6(50.5)$ \\
\hline \multicolumn{3}{|l|}{ DMARDs at the time of additional dose, $\mathrm{n}(\%) \ddagger$} \\
\hline Methotrexate & $30(42.3)$ & $16(59.3)$ \\
\hline TNFi & $27(38.0)$ & $13(48.1)$ \\
\hline Prednisone & $15(21.1)$ & $3(11.1)$ \\
\hline JAKi & $11(15.5)$ & $4(14.8)$ \\
\hline Abatacept & $5(7.0)$ & $0(0)$ \\
\hline Tocilizumab & $5(7.0)$ & $1(3.7)$ \\
\hline Rituximab & $3(4.2)$ & $2(7.4)$ \\
\hline Other & $13(18.3)$ & $2(7.4)$ \\
\hline None & $3(4.2)$ & $0(0)$ \\
\hline $\begin{array}{l}\text { Held DMARD(s) around the time of } \\
\text { additional dose, } n(\%) \S\end{array}$ & 35 (49.3) & 16 (59.3) \\
\hline
\end{tabular}

*One subject provided pre-AddDose and post-AddDose blood samples but did not complete post-AddDose rheumatoid arthritis disease activity assessments. This subject only contributed flow cytometry data and was not included among the 71 subjects in the primary analysis.

tAmong 70 subjects with data on race.

ҒDMARD groups are not mutually exclusive.

$\S$ Among 35 subjects who held DMARDs, 21 held all DMARDs and 14 held at least 1 but not all DMARDs.

DMARDs, disease-modifying antirheumatic drugs; JAKi, Janus kinase inhibitor; RA, rheumatoid arthritis; TNFi, tumour necrosis factor inhibitor.

$\mathrm{T}$ follicular helper cells, age-associated $\mathrm{B}$ cells and plasmablasts-in blood samples obtained pre-AddDose and week 4 post-AddDose. ${ }^{4-6}$ Subject characteristics are shown in table 1. Frequencies of these lymphocyte populations did not significantly differ between the pre-AddDose and post-AddDose timepoints in either subjects who held at least one DMARD $(n=16)$ or subjects who continued all DMARD $(n=11)$ (figure 1$)$.

Limitations of this study include a relatively small number of participants, though the study was adequately powered to detect a difference of $15 \%$ in RA disease activity pre-AddDose versus post-AddDose. The number of participants providing blood for flow cytometry analyses was low, particularly in subgroups by holding versus continuing DMARDs, and results should be interpreted cautiously.

These results suggest that RA disease activity, measured weekly with a validated patient-reported outcome, is stable around the time of an AddDose of COVID-19 vaccine. Lymphocyte subsets of interest in RA were also similar before and after the AddDose, supporting the observation of stable patient-reported RA disease activity. Holding DMARDs was not associated with greater RA disease activity following the AddDose. 
A RADAI-5 weekly among 35 subjects that held DMARDs

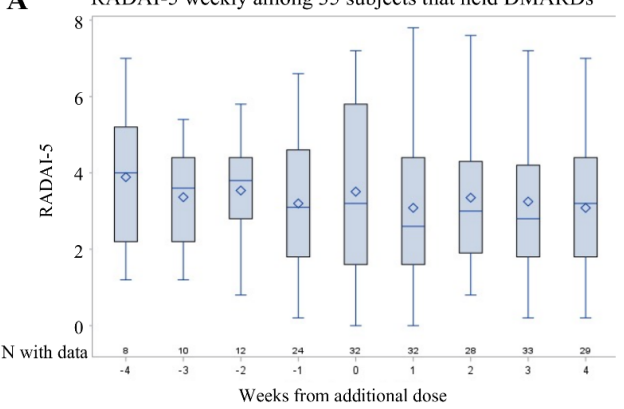

RADAI-5 weekly among 36 subjects that continued DMARDs

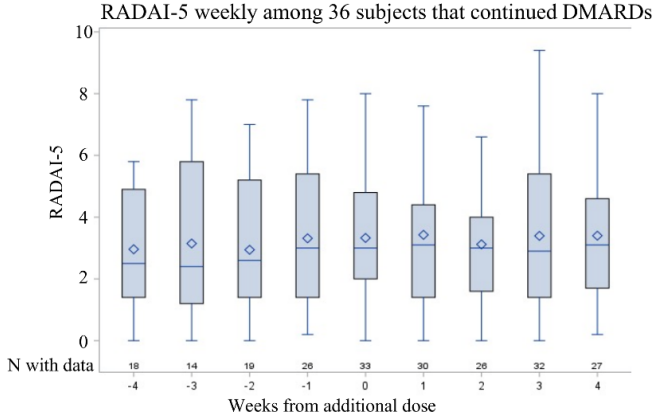

B Lymphocyte subsets among 16 subjects with seropositive RA that held DMARDs
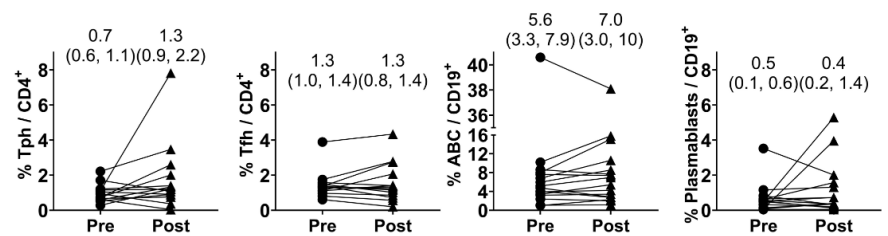

Lymphocyte subsets among 11 subjects with seropositive RA that continued DMARDs
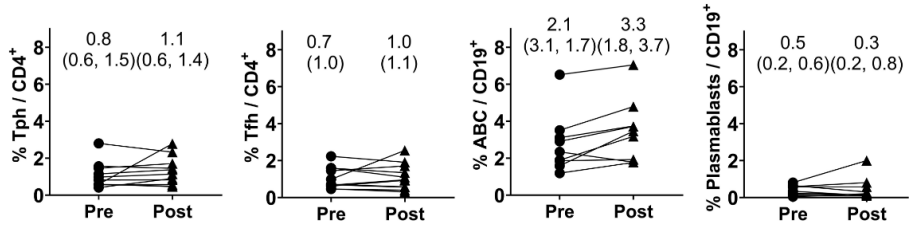

Figure 1 Rheumatoid arthritis (RA) disease activity and lymphocyte subsets among subjects who held at least one disease-modifying antirheumatic drug (DMARD) (top panels) and those who continued all DMARDs (bottom panels) around the time of the additional dose of COVID-19 vaccine. (A) Box-and-whisker plots for Rheumatoid Arthritis Disease Activity Index - 5 (RADAI-5) indicate the median (line), mean (diamond), 25th percentile (bottom of box), 75th percentile (top of box), and minimum and maximum values (whiskers). (B) Lymphocyte populations were gated as follows:

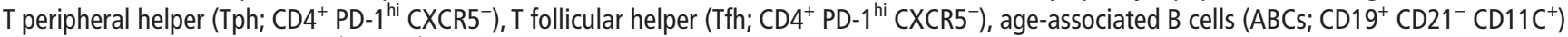
and plasmablasts $\left(C D 19^{+} C D 27^{\text {hi }} \mathrm{CD} 38^{\text {hi }}\right)$. Patients treated with rituximab $(n=3)$ were included in T-cell analyses, but excluded from $B$-cell analyses. Summary data on each panel include median (IQR) \% lymphocyte population pre and post additional dose. Significance testing of flow cytometry data used Wilcoxon paired tests with Bonferroni correction for multiple testing. All comparisons of pre versus post were non-significant ( $p>0.0125$ ).

Sara K Tedeschi $\odot{ }^{1,2}$ Jacklyn Stratton, ${ }^{1}$ Jack Elias Ellrodt, ${ }^{1}$ Mary Grace Whelan, ${ }^{1}$ Keigo Hayashi, ${ }^{1}$ Kazuki Yoshida ${ }^{1,2}$ Lin Chen $\odot{ }^{1}$ Ifeoluwakiisi Adejoorin, ${ }^{1}$ Kathryne Elizabeth Marks, ${ }^{1,2}$ A. Helena Jonsson, ${ }^{1}$ Deepak A Rao $\odot$, $^{1,2}$ Daniel H Solomon ${ }^{1,2}$

'Division of Rheumatology, Inflammation, and Immunity, Brigham and Women's Hospital, Boston, Massachusetts, USA

${ }^{2}$ Department of Medicine, Harvard Medical School, Boston, Massachusetts, USA

Correspondence to Dr Sara K Tedeschi, Division of Rheumatology, Inflammation, and Immunity, Brigham and Women's Hospital, Boston, Massachusetts, USA; stedeschi1@bwh.harvard.edu

\section{Handling editor Josef S Smolen}

Twitter Kazuki Yoshida @kaz_yos and Deepak A Rao @deepakarao

Contributors SKT and DHS were responsible for conceiving the study, overseeing recruitment and data interpretation. SKT drafted the first version of the manuscript. JS, JEE, and MGW were responsible for subject recruitment, conducting study visits, data entry and provided comments on the manuscript. $\mathrm{KH}$ performed data analysis and provided comments on the manuscript. KY provided biostatistical input and provided comments on the manuscript. LC, IA and KEM performed flow cytometry and data analyses and provided comments on the manuscript. AHJ contributed to study design and provided critical feedback on the manuscript. DAR contributed to study design, oversaw flow cytometry analyses and provided critical feedback on the manuscript.

Funding ModernaTx provided support to Brigham and Women's Hospital for an Investigator Sponsored Study and ModernaTx approved the final manuscript, but was not involved with study design, study conduct, data analysis, results interpretation or drafting the manuscript. Additional support for the investigators' time came from the National Institutes of Health (K23 AR075070, L30 AR070514, K23 AR076453, K08 AR072791, P30 AR072577 and P30 AR070253). DAR receives support from the Clinical Scientist Development Award from the Doris Duke Charitable Foundation.

Competing interests ModernaTx provided support for this investigator-sponsored study (PI: DHS) with payments made directly to Brigham and Women's Hospital. SKT: research support to Brigham and Women's Hospital from $\mathrm{NIH}$; consulting fees from NGM Biopharmaceuticals. KY: research support to Brigham and Women's Hospital from NIH; consulting fees from OM1, Inc. AHJ: research support to Brigham and Women's Hospital from Amgen. DAR: research support to Brigham and Women's Hospital from Doris Duke Charitable Foundation, Jansen, Merck, NIH; scientific advisory board member for Bristol Myers Squibb; patent submitted on T peripheral helper cells as a biomarker in autoimmune disease; consulting fees from Jansen. DHS: research support to Brigham and Women's Hospital from ModernaTx, Amgen, Abbvie, CorEvitas, and NIH; royalties from UpToDate for a chapter related to NSAIDs.

Patient consent for publication Not applicable.

Ethics approval This study involves human participants and was approved by Mass General Brigham Institutional Review Board Protocol \# 2021P00763. Participants gave informed consent to participate in the study before taking part.

Provenance and peer review Not commissioned; externally peer reviewed.

Supplemental material This content has been supplied by the author(s). It has not been vetted by BMJ Publishing Group Limited (BMJ) and may not have been peer-reviewed. Any opinions or recommendations discussed are solely those of the author(s) and are not endorsed by BMJ. BMJ disclaims all liability and responsibility arising from any reliance placed on the content. Where the content includes any translated material, BMJ does not warrant the accuracy and reliability of the translations (including but not limited to local regulations, clinical guidelines, terminology, drug names and drug dosages), and is not responsible for any error and/or omissions arising from translation and adaptation or otherwise.

(c) Author(s) (or their employer(s)) 2022. No commercial re-use. See rights and permissions. Published by BMJ.

- Additional supplemental material is published online only. To view, please visit the journal online (http://dx.doi.org/10.1136/annrheumdis-2022-222232).

DAR and DHS are joint senior authors.

\section{Check for updates}

To cite Tedeschi SK, Stratton J, Ellrodt JE, et al. Ann Rheum Dis Epub ahead of print: [please include Day Month Year]. doi:10.1136/annrheumdis-2022-222232

Received 27 January 2022

Accepted 4 February 2022 
Ann Rheum Dis 2022;0:1-3. doi:10.1136/annrheumdis-2022-222232

ORCID iDs

Sara K Tedeschi http://orcid.org/0000-0001-9475-1363

Lin Chen http://orcid.org/0000-0001-5072-4152

Deepak A Rao http://orcid.org/0000-0001-9672-7746

\section{REFERENCES}

1 Centers for Disease Control and Prevention. Covid-19 vaccines for moderately or severely immunocompromised people, 2021. Available: https://www.cdc.gov/ coronavirus/2019-ncov/vaccines/recommendations/immuno.html

2 American College of Rheumatology. Covid-19 vaccine clinical guidance summary for patients with rheumatic and musculoskeletal diseases, 2021. Available: https://www.
rheumatology.org/Portals/O/Files/COVID-19-Vaccine-Clinical-Guidance-RheumaticDiseases-Summary.pdf

3 Leeb BF, Haindl PM, Maktari A, et al. Patient-centered rheumatoid arthritis disease activity assessment by a modified radai. J Rheumatol 2008;35:1294-9.

4 Rao DA, Gurish MF, Marshall JL, et al. Pathologically expanded peripheral T helper cell subset drives B cells in rheumatoid arthritis. Nature 2017:542:110-4.

5 Rubtsov AV, Rubtsova K, Fischer A, et al. Toll-like receptor 7 (tlr7)-driven accumulation of a novel cd11c B-cell population is important for the development of autoimmunity. Blood 2011;118:1305-15.

6 Kerkman PF, Rombouts Y, van der Voort ElH, et al. Circulating plasmablasts/plasmacells as a source of anticitrullinated protein antibodies in patients with rheumatoid arthritis. Ann Rheum Dis 2013;72:1259-63. 